\title{
Gene Expression Profile in the Early Stage of Angiotensin II-induced Cardiac Remodeling: a Time Series Microarray Study in a Mouse Model
}

\author{
Meng-Qiu Dang ${ }^{\mathrm{a}} \quad$ Xue-Chen Zhao ${ }^{\mathrm{a}} \quad$ Song Lai ${ }^{\mathrm{a}} \quad \mathrm{Xia}_{\mathrm{W}} \mathrm{Wang}^{\mathrm{b}} \quad$ Lei Wang $^{\mathrm{a}}$ \\ Yun-Long Zhang ${ }^{a}$ Yang Liu ${ }^{a}$ Xiao-Hong Yua Ying Liu ${ }^{a}$ Hui-Hua Lia,b \\ Yun-Long Xia ${ }^{a}$
}

aDepartment of Cardiology, Institute of Cardiovascular Diseases, First Affiliated Hospital of Dalian Medical University, ${ }^{b}$ Department of Nutrition and Food Hygiene, Center for Prevention and Control of Non-communicable Chronic Diseases, School of Public Health, Dalian Medical University, Dalian, PR China

\section{Key Words}

Early cardiac remodeling • Angiotensin II • Microarray • Gene expression profiles $\bullet$ Serpine1

\begin{abstract}
Background/Aims: Angiotensin II (Ang II) plays a critical role in the cardiac remodeling contributing to heart failure. However, the gene expression profiles induced by Ang II in the early stage of cardiac remodeling remain unknown. Methods: Wild-type male mice (C57BL/6 background, 10-weeek-old) were infused with Ang II (1500 ng/kg/min) for 7 days. Blood pressure was measured. Cardiac function and remodeling were examined by echocardiography, $\mathrm{H} \& \mathrm{E}$ and Masson staining. The time series microarrays were then conducted to detected gene expression profiles. Results: Microarray results identified that 1,489 genes were differentially expressed in the hearts at day 1, 3 and 7 of Ang II injection. These genes were further classified into 26 profiles by hierarchical cluster analysis. Of them, 4 profiles were significant (No. 19, 8, 21 and 22) and contained 904 genes. Gene Ontology showed that these genes mainly participate in metabolic process, oxidation-reduction process, extracellular matrix organization, apoptotic process, immune response, and others. Significant pathways included focal adhesion, ECM-receptor interaction, cytokine-cytokine receptor interaction, MAPK and insulin signaling pathways, which were known to play important roles in Ang II-induced cardiac remodeling. Moreover, gene co-expression networks analysis suggested that serine/ cysteine peptidase inhibitor, member 1 (Serpine1, also known as PAI-1) localized in the core of the network. Conclusions: Our results indicate that many genes are mainly involved in metabolism, inflammation, cardiac fibrosis and hypertrophy. Serpine1 may play a central role in the development of Ang II-induced cardiac remodeling at the early stage.

Copyright (C) 2015 S. Karger AG, Basel

M.-Q. Dang, X.-C. Zhao and S. Lai contributed equally to this work.

Yun-Long Xia or Hui-Hua Li

Department of Cardiology, Institute of Cardiovascular Diseases, First Affiliated Hospital of Dalian Medical University, Dalian 116011 (China)

E-Mail yunlong_xia@126.com, hhli1995@yahoo.com
\end{abstract}

KARGER 125 


\section{Introduction}

The renin-angiotensin system (RAS) plays a crucial role in cardiovascular homoeostasis via the activity of its effector, angiotensin II (Ang II) [1, 2]. Ang II mediates a range of physiological and pathophysiological actions by interacting with its receptor (Ang II type 1 receptor, AT1R) [1]. Extensive studies have identified the role of Ang II in regulation of blood pressure. Despite the successful use of angiotensin-converting enzyme inhibitor (ACEIs) and angiotensin receptor blocker (ARB) in the clinical practice, growing evidence has shown that Ang II might contribute to cardiovascular diseases by other mechanisms, independent actions of in lowering blood pressure.

Cardiac remodeling is a major risk factor for cardiovascular diseases [3]. Remodeling normally occurs in the form of hypertrophy, apoptosis and fibrosis, eventually leading to the development of pathological cardiac hypertrophy and heart failure [4]. Chronic increases in cardiac Ang II level have been reported in both experimental models and clinical studies during the development of heart failure. Inhibition of RAS pathway is effective for cardiac remodeling and heart failure, independently of the reduction in systemic blood pressure [5]. Several studies have demonstrated that Ang II-mediated intracellular signal transductions contribute to the progress of cardiac remodeling, including production of reactive oxygen species (ROS), activation of receptor and non-receptor tyrosine kinases and serine/threonine kinases, such as mitogen-activated protein kinase family (ERK, JNK and p38 MAPK), AKT/ mTOR/ p70S6K and various PKC isoforms [6-10]. Studies also reveal that Ang II-mediated effects in the heart are accompanied by alterations in gene expression profile [11]. However, the molecular mechanistic insight by which Ang II contributes to early cardiac remodeling remains obscure.

In the present study, we used time series microarrays to analyze the gene expression profiles in the heart at days 1, 3, and 7 after Ang II infusion. Our results showed that 1489 genes were significantly changed in Ang II-infused heart at these time points. The genes were classified into 26 clusters based on the gene expression pattern. Four profiles (No. 19, 8, 21 and 22) contained 904 genes were significantly changed. Finally, gene co-expression networks analysis identified serine/cysteine peptidase inhibitor, member 1 (Serpine1, also known PAI-1) as a core gene, which may play a central role in the early stage of Ang IIinduced cardiac remodeling.

\section{Materials and Methods}

\section{Animal model}

Wild-type male mice (C57BL/6, 10-week-old, n=6 per group) were infused with Ang II at $1500 \mathrm{ng} / \mathrm{kg} /$ min in Ringer's solution with osmotic pumps (Alzet Model 1007D; USA) for 1, 3, and 7 days as described [12-18]. Systolic blood pressure was monitored by the tail-cuff method. Animals were anesthetized with isoflurane (1.5\%), and underwent M-mode echocardiography at each time point using a $30 \mathrm{MHz}$ probe (Vevo 770 system; VisualSonics, Toronto, Ontario, Canada). All procedures were performed in accordance with the Animal Care and Use Committee of Capital Medical University. The investigation conformed to the Guide for the Care and Use of Laboratory Animals published by the U.S. National Institutes of Health (NIH Publication No.85-23, revised 1996).

\section{Histological analysis}

The hearts were fixed in phosphate-buffered 4\% formalin ( $\mathrm{pH} 7.4$ ) for $24 \mathrm{~h}$ and then embedded in paraffin. The heart sections (5 $\mu \mathrm{m}$ ) were examined by H\&E, Masson's trichrome, and WGA staining [12-18]. Sections were also incubated with a Mac-2 (a marker for macrophages) antibody (Santa Cruz, CA, USA) as described [13]. Images were viewed and captured using a Nikon Labophot 2 microscope (Nikon, Tokyo, Japan).

Microarray assay and comprehensive bioinformatics analysis

Mice were euthanized by an overdose of pentobarbital (100 mg/kg, intraperitoneal injection) at day 1, 3 and 7 post Ang II infusion. Hearts ( $n=3$ per group) were removed and then frozen in liquid nitrogen KARGER 
Table 1. Sequences of the primers used in the SYBR-green-based qRTPCR validation

\begin{tabular}{lll}
\hline Gene symbol & \multicolumn{1}{c}{ Forward primer (5' to 3') } & \multicolumn{1}{c}{ Reverse primer (5' to 3') } \\
\hline Pank4 & CCAGCCCTTTGCTTATGGGA & TAGTGCGAGGCCGTTTCTT \\
Ccng1 & AATGAAGGTACAGGCGAAGCA & TCAGTCGCTTTCACAGCCAA \\
Hspb1 & TCACCCGGAAATACACGCTC & GGCCTCGAAAGTAACCGGAA \\
Ccl6 44 & CCTTGGCCACCACTCCTAAT & CCGAAGCAGTTGTCCCTTCT \\
Ccl7 & GCCGGGCATCATCTTTATCA & AGATCTTGGGCCTTGCTTCA \\
S100a8 & GATCTCTGCCACGCTTCTGT & GCATTGGGCCCATCTGGTTG \\
Ipo4 & GTCCTCAGTTTGTGCAGAATATAAA & TTTGTGAGATGCCACACCCA \\
Nop58 & CAGGCAGAGACTGCTGTACC & GCGAACAGAGCAGCATTACG \\
Utp23 & AGCACAGCAGTTCCTAGTCC & GATGTGCTCTGCTCCCAACT \\
Smad1 & CGAGCCGGCGCTAACTG & CCTCATCCACCATGGACAG \\
Tgfb2 & CGAGGCGAGATTTGCAGGTAT & GCTTCTTGGTCTGCGGTTTG \\
Cxcl1 & ACCGAAGTCATAGCCACACTC & CTCCGTTACTTGGGGACACC \\
Hsp90aa1 & TGTTCATTCAGCCACGATGC & ACTGGGCAATTTCTGCCTGA \\
Timp1 & AGTGATTTCCCCGCCAACTC & GGTATCTGCTCTGGTGTGTCT \\
Ier3 & CTCGAGTGGTCCGGCG & CTGGCAGAAGATGATGGCGA \\
Serpine1 & GCACAACCCGACAGAGACAA & ATGAAGGCGTCTCTTCCCAC \\
Ccr2 & GGAGCCATACCTGTAAATGCC & TGGTGAATCCAATGCCCTCT \\
Col4a2 & CGGCGTAATCTCAAAAGGCG & GGCCTCTGCTTCCTTTCTGT \\
\hline
\end{tabular}

[12-15]. Total RNA was isolated with TRIzol (Invitrogen) from hearts collected at day 1, 3 and 7 according to manufacturer's instructions. Gene expression profiling was performed using mouse Genome 4302.0 array according to the manufacturer's instructions (Affymetrix, Inc., Santa Clara, CA) [14, 17, 19]. Fifteen micrograms of biotin-labeled complementary RNA was fractionated and hybridized to Affymetrix GeneChip. Microarray data was analyzed as described previously [14,17,19]. A comprehensive bioinformatics analysis was used to enrich the dataset for genes associated with Ang II-induced heart remodeling, including gene ontology (GO), pathway, series test of clustering, and gene co-expression network [19]. The details of gene expression data are available at the GEO website: http://www.ncbi.nlm.nih.gov/geo/ (accession number GSE59437).

Validation of microarray data by qPCR analysis

Quantitative real-time PCR (qPCR) was used to verify the differential expression of 19 selected genes that were detected by microarray. These genes included Pank4, Ccng1, Hspb1, CD44, Ccl6, Ccl7, S100a8, Ipo4, Nop58, Utp23, Smad1, Tgfb2, Cxcl1, Hsp90aa1, Timp1, Ier3, Serpine1, Ccr2 and Col4a2. The qPCR analysis was performed with an iCycler IQ system (Bio-Rad, USA) as described [13-15, 19]. The primers used were listed in Table 1 . The cycling conditions consisted an initial, single cycle of 5 min at $95^{\circ} \mathrm{C}$, followed by $40 \mathrm{cycles}$ of $30 \mathrm{sec}$ at $95^{\circ} \mathrm{C}, 30 \mathrm{sec}$ at $54^{\circ} \mathrm{C}, 15 \mathrm{sec}$ at $72^{\circ} \mathrm{C}$, and fluorescence acquisition at $83^{\circ} \mathrm{C}$ for $1 \mathrm{sec}$. The gene expression levels were quantified relative to the expression of GAPDH.

Statistical analysis

All values were presented as mean \pm S. E. M. Statistical differences between groups were analyzed by the non-parametric tests (Mann-Whitney) or by the parametric test one-way analysis of variance (ANOVA) followed by the Tukey-Kramer test for group differences. $P<0.05$ was considered significant.

\section{Results}

Ang II increase blood pressure and cardiac remodeling in mice

To investigate the alteration of gene expression profiles in Ang II-induced cardiac remodeling, wild-type male mice were infused with Ang II at1500 ng/kg/min for 7 days. Ang II infusion led to a significant increase over the baseline value in systolic blood pressure as early as day 1, which progressively increased till day 7 post Ang II infusion (Fig. 1A). Moreover, inflammatory cells accumulated in the myocadirum was significantly increased at day 1 after Ang II infusion and reached the peak level at day 3. Elevated collagen deposition was observed at day 3 and then further increased at day 7. Up-regulation in cross-sectional 
A

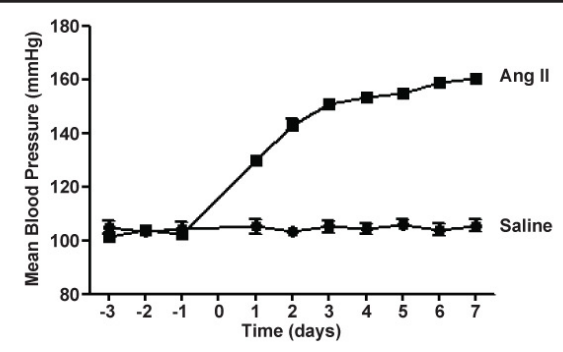

B
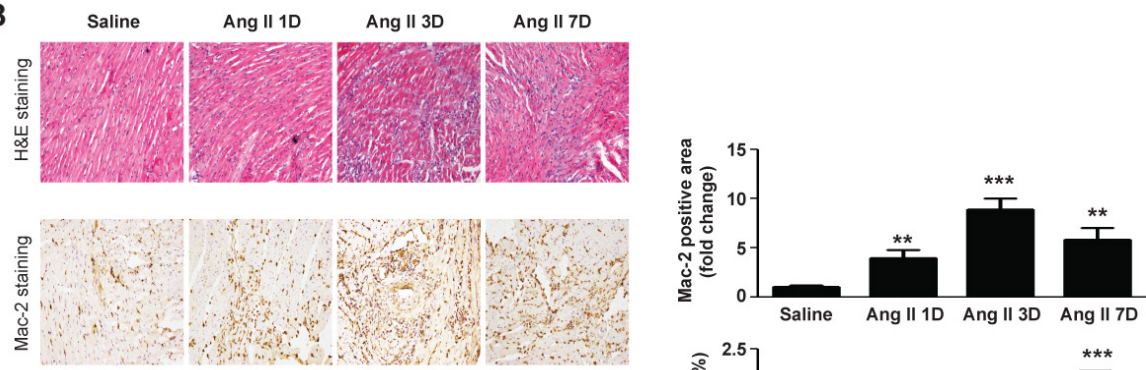

C
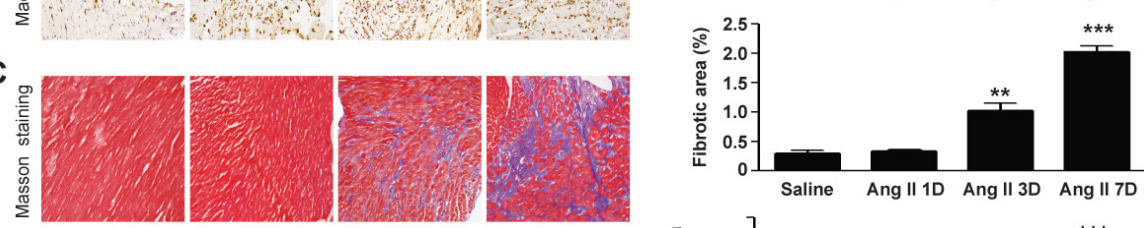

D
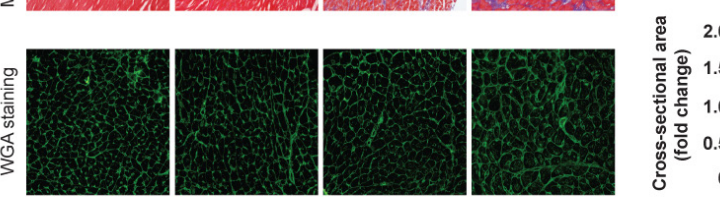

Saline Ang II 1D Ang II 3D Ang II 7D

E
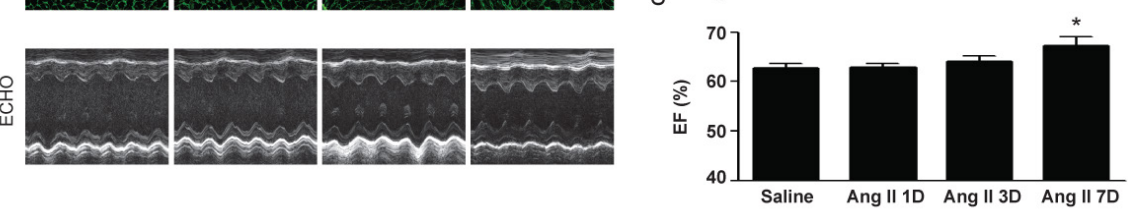

Fig. 1. Effects of Ang II infusion on blood pressure and cardiac remodeling in mice. (A) Wild-type mice were injected with Ang II (1500 ng/kg/min) for 1, 3 and 7 days. Systolic blood pressure was measured by the tail cuff method ( $n=8$ to 10 per group). (B) Representative H\&E and immunohistochemical staining of mac-2 in the heart sections (magnification, x200). Bar graphs show quantification of mac-2 positive area. (C) Representative Masson trichrome staining (blue) of heart sections analyzed for fibrotic areas (magnification, x100). Bar graphs show quantification of fibrotic areas. (D) Wheat germ agglutinin (WGA) staining of heart sections indicates Ang II-induced modest cardiomyocyte hypertrophy (magnification, x400). Bar graphs show quantification of cross-sectional area. (E) Representative two-dimensional guided M-mode images of the LV demonstrate Ang II infusion increased wall thickness. Bar graphs show quantification of EF\% ( $\mathrm{n}=8$ to 10 per group). Data are expressed as mean \pm SEM. ${ }^{*} \mathrm{P}<0.05 ;{ }^{* *} \mathrm{P}<0.01 ;{ }^{* * *} \mathrm{P}<0.001$ vs saline.

area of cardiomyocytes was observed at day 7 (Fig. 1B-D). Echocardiographic measurement revealed that Ang II infusion also markedly increased cardiac function characterized by increased EF\% at day 7 (Fig. 1E). These results suggest that acute cardiac remodeling induced by Ang II infusion might start from day 3.

\section{Microarray assay in Ang II-infused hearts}

To profile the gene expression in Ang II-treated heart, tissues were isolated from the mice at day 1, 3, and 7 post Ang II infusion. The time series microarrays ( $\mathrm{n}=3$ per time point) were performed. A total of 1489 genes were differentially expressed in Ang II-treated hearts at least at one time point compared to saline controls $(\mathrm{P}<0.05)$. Of them, 862 genes were 


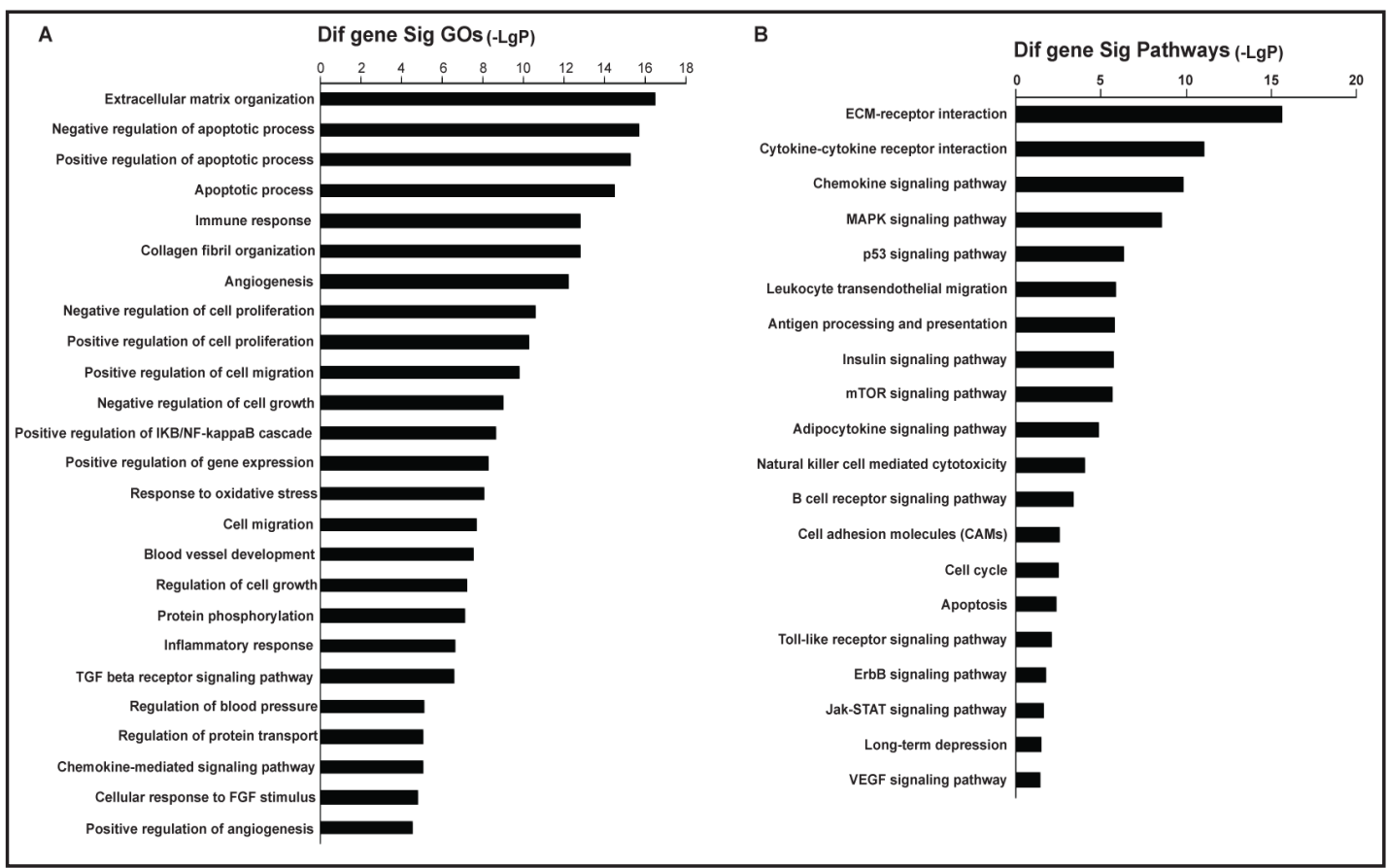

Fig. 2. GOs terms and KEGG pathways. (A) GO category analysis based on biological process for differentially expressed genes. LgP is the logarithm of $P$-value. (B) KEGG pathway analysis for the differentially expressed genes in the Ang II-treated heart.

Fig. 3. Identification of significant gene expression profiles by hierarchical clustering analysis in Ang II-treated hearts. Total 1,489 differentially expressed genes were classified into 26 profiles. Each box represents a model expression profile. The number in the top represents the profile ID and p-value. Four gene expression patterns (No. 19, 8, 21 and 22) showed statistically significant $p$-values $(p<0.00001)$ (red colored boxes). The horizontal axis represents time points, and the vertical axis shows the time series of gene expression levels for the gene after Log normalized transformation.

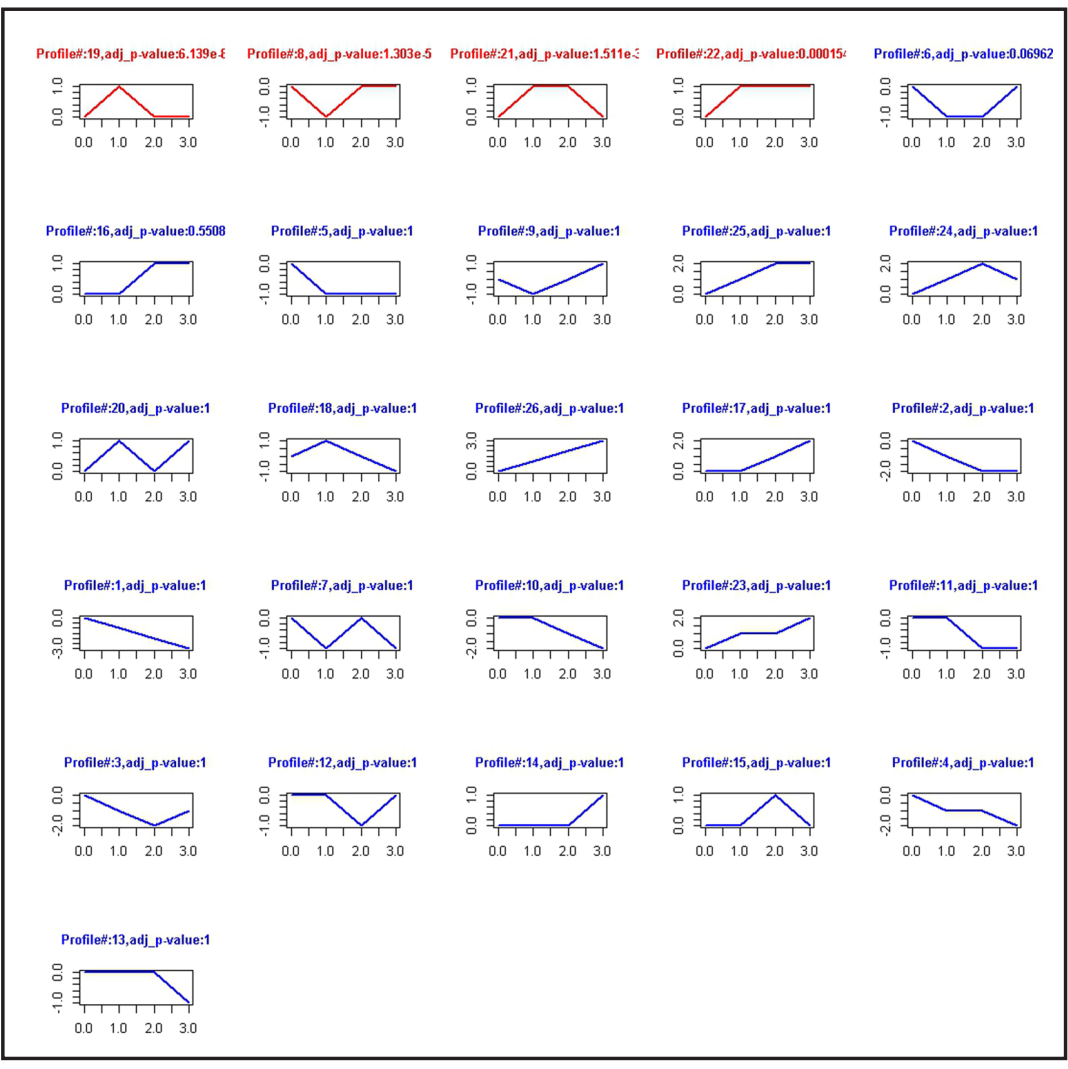

significantly up-regulated whereas 672 genes were down-regulated at day 1; 909 genes markedly were up-regulated whereas 580 genes were down-regulated at day 3; 931 genes were increased whereas 558 genes were decreased at day 7 . 


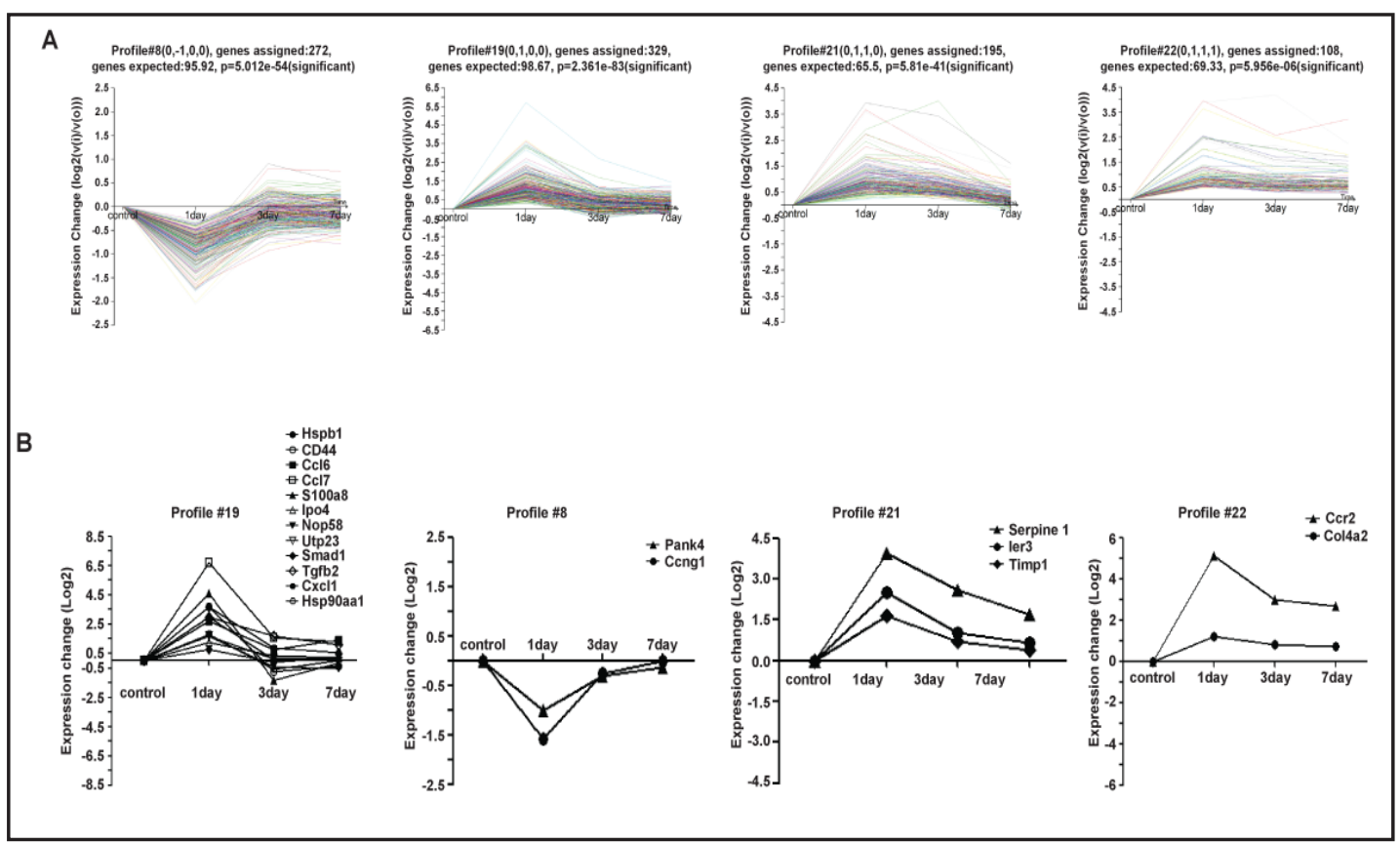

Fig. 4. Confirmation of microarray data by qPCR analysis in Ang II-treated hearts. The expression levels of 19 differentially genes from significant profiles were confirmed by qPCR analysis, including No. 19 (Hspb1, CD44, Ccl6, Ccl7, S100a8, Ipo4, Nop58, Utp23, Smad1, Tgfb2, Cxcl1 and Hsp90aa1); No. 8 (Pank4, Ccng1); No. 21 (Timp1, Ier3 and Serpine 1), and No. 22 (Ccr2 and Col4a2). GAPDH as an internal control. Data are presented as mean \pm SEM ( $n=4-6$ per group).

\section{GO and pathway analysis}

During Ang II infusion, the most significantly GO terms $(P<0.001)$ included metabolic process, oxidation-reduction process, transport, extracellular matrix organization, apoptotic process, immune response, angiogenesis, and chemotaxis, etc (Fig. 2A). Furthermore, pathway analysis identified 66 pathways that were significantly altered in Ang II treated hearts $(P<0.05)$. These pathways involved in focal adhesion, ECM-receptor interaction, cytokine-cytokine receptor interaction, chemokine, regulation of actin cytoskeleton, MAPK p53, insulin/mTOR, adipocytokine, neurotrophin signaling pathway (Fig. 2B), suggesting that GO terms and pathways may play critical roles in the development of Ang II-induced cardiac remodeling.

\section{Cluster analysis}

To determine the gene expression patterns, we next performed hierarchical cluster analysis. The total 1,489 genes were classified into 26 profiles (Fig. 3). Among them, 4 significant profiles (including No. 19, 8, 21 and 22) contained total 904 genes were identified. According to ascending $p$-values $(2.361 \mathrm{E}-83)$, profile 19 was considered to be the most important pattern. The profile 19 contained 329 genes and showed increased expression at day 1 and then markedly returned to the control level at day 3 after Ang II infusion, while genes in profile 8 (272 genes) were down-regulated at day 1 and then went back to the baseline value. Gene expressions in profiles 21 and 22 were peaked at day 1 and then gradually decreased at day 3 and 7.

\section{Verification of gene expression by qPCR analysis}

In order to validate the microrarray data, we selected 19 genes from 4 significant profiles, including No.19 (Hspb1, CD44, Ccl6, Ccl7, S100a8, Ipo4, Nop58, Utp23, Smad1, Tgfb2, Cxcl1 and Hsp90aa1), No. 8 (Pank4 and Ccng1), No. 21 (Timp1, Ier3 and Serpine 1) and No. 22 (Ccr2 and Col4a2), and performed qPCR at different time points. Fig. 4 showed 


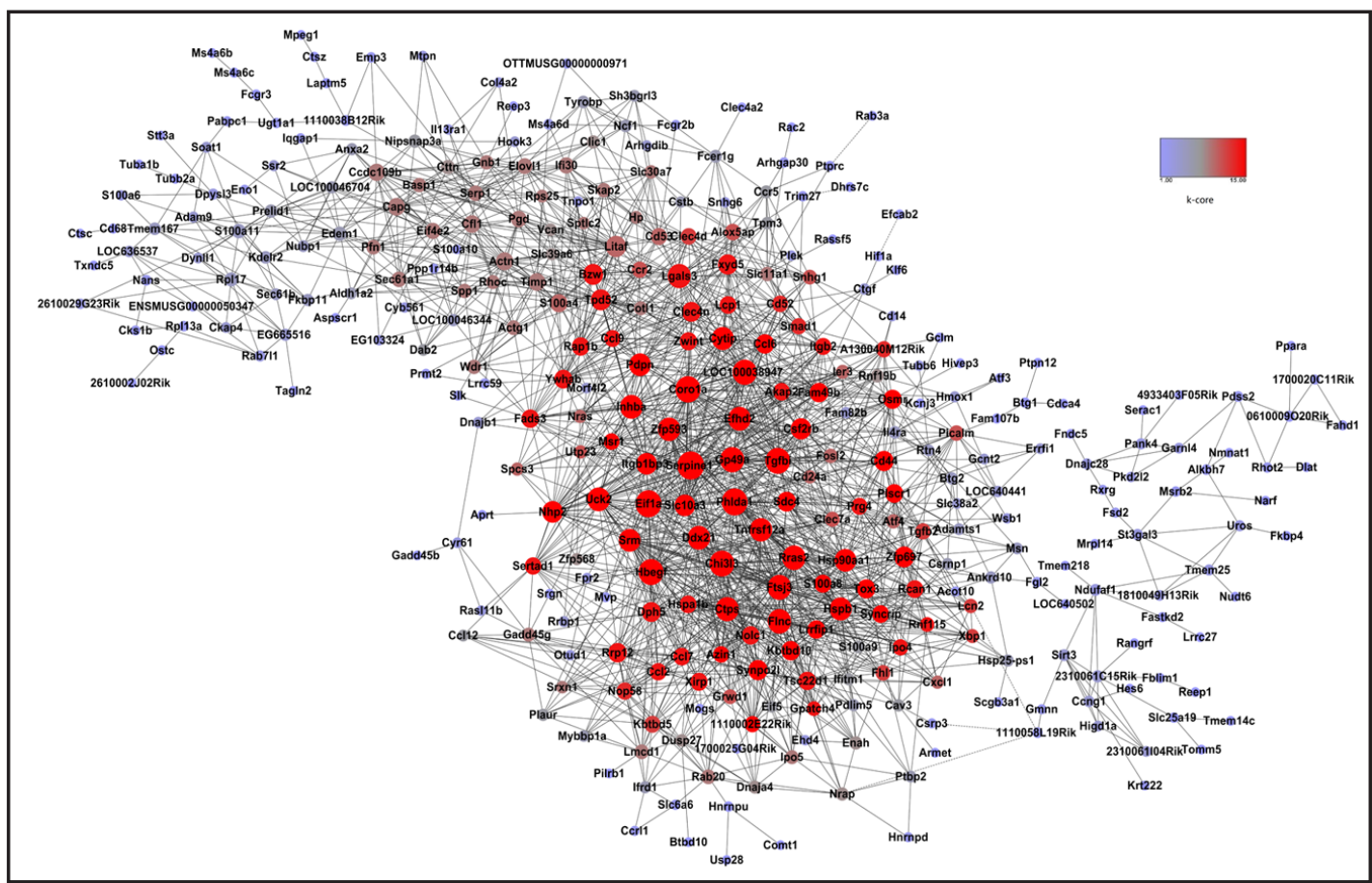

Fig. 5. Analysis of gene co-expression network in Ang II-treated hearts. Total 904 genes in 4 significant profiles (No. 19, 8, 21, and 22) were analyzed by gene co-expression network with the k-core algorithm. Cycle nodes represent genes, and edges between two nodes represent interactions between genes which were quantified by degree. Genes with higher k-core values are considered to centralize in the network and have a stronger capacity of regulating adjacent genes.

Table 2.19 genes identified by gene co-expression network with k-core algorithm

\begin{tabular}{|c|c|c|c|c|c|}
\hline Profile & $\begin{array}{c}\text { Gene } \\
\text { symbol }\end{array}$ & Gene Title & $\begin{array}{l}\text { Clustering } \\
\text { Coefficient }\end{array}$ & Degree & $\begin{array}{c}\mathrm{K}- \\
\text { core }\end{array}$ \\
\hline 8 & Pank4 & pantothenate kinase 4 & 0.3 & 5 & 3 \\
\hline 8 & Cong1 & cyclin G1 & 0.53333333 & 6 & 4 \\
\hline 19 & Hspb1 & heat shock protein 1 & 0.44950213 & 38 & 15 \\
\hline 19 & $\mathrm{Cd} 44$ & CD44 antigen & 0.45057471 & 30 & 15 \\
\hline 19 & Ccl6 & chemokine (C-C motif) ligand 6 & 0.56084656 & 28 & 15 \\
\hline 19 & Ccl7 & chemokine ( $\mathrm{C}-\mathrm{C}$ motif) ligand 7 & 0.63241107 & 23 & 14 \\
\hline 19 & S100a8 & S100 calcium binding protein A8 & 0.54710145 & 24 & 15 \\
\hline 19 & Ipo4 & importin 4 & 0.64327485 & 19 & 14 \\
\hline 19 & Nop58 & NOP58 ribonucleoprotein homolog & 0.59307359 & 22 & 13 \\
\hline 19 & Utp23 & UTP23,small subunit (SSU) processome component, homolog & 0.8 & 11 & 11 \\
\hline 19 & Smad1 & MAD homolog 1 (Drosophila) & 0.57309942 & 19 & 13 \\
\hline 19 & Tgfb2 & transforming growth factor, beta 2 & 0.38596491 & 19 & 12 \\
\hline 19 & Cxcl1 & chemokine ( $\mathrm{C}-\mathrm{X}-\mathrm{C}$ motif) ligand 1 & 0.65454545 & 11 & 10 \\
\hline 19 & Hsp90aa1 & heat shock protein 90, alpha (cytosolic), class A member 1 & 0.49099099 & 37 & 15 \\
\hline 21 & Timp1 & tissue inhibitor of metalloproteinase 1 & 0.34782609 & 23 & 9 \\
\hline 21 & Ier3 & immediate early response 3 & 0.52564103 & 13 & 10 \\
\hline 21 & Serpine1 & serine (or cysteine) peptidase inhibitor, clade E, member 1 & 0.40045249 & 52 & 15 \\
\hline 22 & Ccr2 & chemokine ( $\mathrm{C}-\mathrm{C}$ motif) receptor 2 & 0.56725146 & 19 & 11 \\
\hline 22 & Col4a2 & collagen, type IV, alpha 2 & 0.33333333 & 3 & 3 \\
\hline
\end{tabular}

the fold changes detected by microarray and qPCR, which are comparable with each other.

Gene co-expression network analysis

To determine which genes that may play key roles in the early cardiac remodeling induced by Ang II, total 904 genes in 4 significant profiles were analyzed by gene co-expression 
networks with the k-core algorithm (Fig. 5). A total of 337 genes from these profiles were selected and analyzed. Based on the K-core value (15), clustering coefficient (0.40), serine/ cysteine peptidase inhibitor, member 1 (Serpine1) appeared at the center of the gene network, which directly regulated 52 neighboring genes. Furthermore, Serpine1 was shown to be involved in regulation of Tgfbi (transforming growth factor, beta induced), Tnfrsf12a (tumor necrosis factor receptor superfamily, member 12a), and Sertad1 (SERTA domain containing 1) in this network. Thus, serpine1 may play a central role in the development of early cardiac remodeling.

\section{Discussion}

In this study, we examined Ang II-treated hearts and made several novel findings. First, Ang II-induced cardiac remodeling (including fibrosis and cardiomyocyte hypertrophy) started at day 3 post infusion. Second, microarrarys showed that a total of 1,489 genes were markedly changed in the heart at day 1, 3, and 7 after Ang II infusion, which were involved in several biological processes including metabolism, oxidation-reduction, extracellular matrix organization, apoptosis and inflammation. Third, gene co-expression network analysis further identified one core gene Serpine1 (also known PAI-1) that may play a critical role in the development of acute cardiac remodeling.

Cardiac hypertrophy, fibrosis, inflammation and apoptosis are the hall markers in the development of cardiac remodeling [20-22], which is mediated by various signaling pathways or mediators, including TGF- $\beta 1 /$ Smad, Rho/Rho kinase, MAPK, mTOR, Jak-STAT, ROS, growth factors, cytokines, and other stress molecules [4, 23]. Apart from them, Ang II-mediated signaling is considered to play a critical role in this process. The binding of Ang II to the AT1 receptor promotes the activation of G-protein-derived second messengers, protein kinases and small G-proteins, promoting cardiac remodeling [1, 24]. However, in the early stage of cardiac remodeling, the alternations in gene expression induced by Ang II remain unclear. Recently, microarray has been wildly used to investigate the global gene expression profiles and has identified many genes that are important for Ang II-induced actions in vascular, kidney, smooth muscle cells, cardiac fibroblasts and cardiomyocytes $[11,25,26]$. In this study, we performed time series microarrays to identify significant differentially expressed genes, GO terms, and KEGG pathways. Our data revealed that total 1489 genes were differentially expressed in the Ang II-infused hearts as compared with saline-treated group. Importantly, many gene expression patterns generated by us were consistent with Schwartz's data [11]. Moreover, we found that Ang II is capable of inducing a wide range of biological processes, including metabolic process, extracellular matrix organization, apoptotic process, immune response, MAPK, insulin/mTOR and others (Fig. 2). Some of these pathways contribute to the process of cardiac fibrosis, inflammation, and hypertrophy [27-29].

Although the mechanisms of Ang II to induce cardiac remodeling have been widely explored, the central genes that contribute to this process remain unknown. In this study, our microarray data revealed that the family of collagen, IGF1, TGFb2, EGFR, CCR, CXCR, CCL, ATF4, FOS, EIF4e, PIK3, SOCS3, etc were differentially expressed, which are known to play important roles in regulating inflammation, fibrosis, hypertrophy and apoptosis [4, 21, 23]. By using clustering and gene co-expression network analysis, we found that Serpine1 (also known as plasminogen activator inhibitor type 1, PAI-1) was localized in core of the gene network, and may play a central role in the progression of Ang II-induced cardiac remodeling (Table 2). Accumulating evidence indicates that Serpine1 can act as 'bait' for many proteolytic factors, including PSMA3 (proteasome subunit alpha type 3), PSMB1 (proteasome subunit, beta type 1), PLAT (plasminogen activator tissue), PLAU (plasminogen activator urokinase) and PLAUR (plasminogen activator, urokinase receptor). Moreover, Serpine1 is the principal inhibitor of tissue plasminogen activator (tPA) and urokinase ( $\mathrm{UPA}$ ), which are primarily responsible for the conversion of plasminogen to plamsmin, leading to fibrinolysis [30]. By inhibiting of tPA and uPA, Serpine 1 contributes to collagen degradation, stromal remodeling, fibrinolysis and 
inflammation [31]. Some epidemiological studies have demonstrated that Serpine1 could be an important risk factor for the initiation and recurrence of cardiovascular diseases [30, 32]. Elevations in the plasma level of Serpine1 have been reported in patients with atherosclerosis and myocardial infarction, and plasma levels are positively correlated with the disease progress [30, 32]. In the present study, we have indicated the gene expression of Serpine1 is up-regulated in the heart after Ang II infusion; however, the cellular source of Serpine1 remains unknown. Serpine1 is mainly localized in smooth muscle, adipocyte, placenta and cardiomyocytes [30, 32-34]. Thus cardiomycoytes could be the primary source of Serpine1. In addition to cardiomycoytes, we have shown that a great number of inflammatory cells accumulated in the heart, inflammatory cells could be an additional cellular source of local Serpine 1 and amplification the process of remodeling, which needs further investigation.

In conclusion, we investigated Ang II-mediated molecular events associated with the development of early cardiac remodeling by using microarrays in a mouse model. We found that total 1,489 genes were differently expressed in the heart at day 1, 3, and 7 after Ang II infusion. These genes were involved in several biological functions. Importantly, gene coexpression network analysis indicated that Serpine1 may play a central role in the formation of acute cardiac remodeling. However, it will be important to determine the mechanism for Ang II to upregulate the expression of Serpine1 in the heart; the role of Serpine1 in modulating Ang II-induced cardiac remodeling; and if Serpine1 could be a novel therapeutic target for cardiac injury.

\section{Acknowledgements}

This study was supported by grants from the 973 program (No. 2012CB517802), National Natural Science Foundation of China (81330003, 81025001), and Chang Jiang Scholar Program.

\section{References}

1 Higuchi S, Ohtsu H, Suzuki H, Shirai H, Frank GD, Eguchi S: Angiotensin ii signal transduction through the at1 receptor: Novel insights into mechanisms and pathophysiology. Clin Sci (Lond) 2007;112:417-428.

$\checkmark 2$ Unger T: The role of the renin-angiotensin system in the development of cardiovascular disease. Am J Cardiol 2002;89:3A-9A; discussion 10A.

-3 Cohn JN, Ferrari R, Sharpe N: Cardiac remodeling--concepts and clinical implications: A consensus paper from an international forum on cardiac remodeling. Behalf of an international forum on cardiac remodeling. J Am Coll Cardiol 2000;35:569-582.

4 Hill JA, Olson EN: Cardiac plasticity. N Engl J Med 2008;358:1370-1380.

$>5$ Levy BI: Can angiotensin ii type 2 receptors have deleterious effects in cardiovascular disease? Implications for therapeutic blockade of the renin-angiotensin system. Circulation 2004;109:8-13.

-6 Griendling KK, Sorescu D, Ushio-Fukai M: Nad(p)h oxidase: Role in cardiovascular biology and disease. Circ Res 2000;86:494-501.

7 Hunyady L, Catt KJ: Pleiotropic at1 receptor signaling pathways mediating physiological and pathogenic actions of angiotensin ii. Mol Endocrinol 2006;20:953-970.

8 Marrero MB, Fulton D, Stepp D, Stern DM: Angiotensin ii-induced insulin resistance and protein tyrosine phosphatases. Arterioscler Thromb Vasc Biol 2004;24:2009-2013.

-9 Schmitz U, Ishida T, Ishida M, Surapisitchat J, Hasham MI, Pelech S, Berk BC: Angiotensin ii stimulates p21activated kinase in vascular smooth muscle cells: Role in activation of jnk. Circ Res 1998;82:1272-1278.

10 Suzuki H, Motley ED, Frank GD, Utsunomiya H, Eguchi S: Recent progress in signal transduction research of the angiotensin ii type-1 receptor: Protein kinases, vascular dysfunction and structural requirement. Curr Med Chem Cardiovasc Hematol Agents 2005;3:305-322. 
11 Schwartz F, Duka A, Duka I, Cui J, Gavras H: Novel targets of ang ii regulation in mouse heart identified by serial analysis of gene expression. Am J Physiol Heart Circ Physiol 2004;287:H1957-1966.

-12 Jiang HM, Wang HX, Yang H, Zeng XJ, Tang CS, Du J, Li HH: Role for granulocyte colony stimulating factor in angiotensin ii-induced neutrophil recruitment and cardiac fibrosis in mice. Am J Hypertens 2013;26:12241233.

13 Wang L, Li YL, Zhang CC, Cui W, Wang X, Xia Y, Du J, Li HH: Inhibition of toll-like receptor 2 reduces cardiac fibrosis by attenuating macrophage-mediated inflammation. Cardiovasc Res 2014;101:383-392.

14 Wu Y, Li Y, Zhang C, A X, Wang Y, Cui W, Li H, Du J: S100a8/a9 released by cd11b+gr1+ neutrophils activates cardiac fibroblasts to initiate angiotensin ii-induced cardiac inflammation and injury. Hypertension 2014;63:1241-1250.

15 Zhang C, Li Y, Wang C, Wu Y, Cui W, Miwa T, Sato S, Li H, Song WC, Du J: Complement 5a receptor mediates angiotensin ii-induced cardiac inflammation and remodeling. Arterioscler Thromb Vasc Biol 2014;34:1240-1248.

16 Wang HX, Yang H, Han QY, Li N, Jiang X, Tian C, Du J, Li HH: Nadph oxidases mediate a cellular "memory" of angiotensin ii stress in hypertensive cardiac hypertrophy. Free Radic Biol Med 2013;65:897-907.

17 Yang D, Zeng Y, Tian C, Liu J, Guo SB, Zheng YH, Li HH: Transcriptomic analysis of mild hypothermiadependent alterations during endothelial reperfusion injury. Cell Physiol Biochem 2010;25:605-614.

18 Yang K, Zhang TP, Tian C, Jia LX, Du J, Li HH: Carboxyl terminus of heat shock protein 70-interacting protein inhibits angiotensin ii-induced cardiac remodeling. Am J Hypertens 2012;25:994-1001.

19 Zhang JS, Zhang YL, Wang HX, Xia YL, Wang L, Jiang YN, Li HH, Liu Y: Identification of genes related to the early stage of angiotensin ii-induced acute renal injury by microarray and integrated gene network analysis. Cell Physiol Biochem 2014;34:1137-1151.

20 Frey N, Katus HA, Olson EN, Hill JA: Hypertrophy of the heart: A new therapeutic target? Circulation 2004;109:1580-1589.

-21 Jia L, Li Y, Xiao C, Du J: Angiotensin ii induces inflammation leading to cardiac remodeling. Front Biosci (Landmark Ed) 2012;17:221-231.

-22 Manabe I, Shindo T, Nagai R: Gene expression in fibroblasts and fibrosis: Involvement in cardiac hypertrophy. Circ Res 2002;91:1103-1113.

23 Frey N, Olson EN: Cardiac hypertrophy: The good, the bad, and the ugly. Annu Rev Physiol 2003;65:45-79.

24 Mehta PK, Griendling KK: Angiotensin ii cell signaling: Physiological and pathological effects in the cardiovascular system. Am J Physiol Cell Physiol 2007;292:C82-97.

25 Madhur MS, Lob HE, McCann LA, Iwakura Y, Blinder Y, Guzik TJ, Harrison DG: Interleukin 17 promotes angiotensin ii-induced hypertension and vascular dysfunction. Hypertension 2010;55:500-507.

-26 Makhanova NA, Crowley SD, Griffiths RC, Coffman TM: Gene expression profiles linked to at1 angiotensin receptors in the kidney. Physiol Genomics 2010;42A:211-218.

27 Berk BC, Fujiwara K, Lehoux S: Ecm remodeling in hypertensive heart disease. J Clin Invest 2007;117:568575.

28 Li YZ, Lu DY, Tan WQ Wang JX, Li PF: P53 initiates apoptosis by transcriptionally targeting the antiapoptotic protein arc. Mol Cell Biol 2008;28:564-574.

29 Thum T, Gross C, Fiedler J, Fischer T, Kissler S, Bussen M, Galuppo P, Just S, Rottbauer W, Frantz S, Castoldi M, Soutschek J, Koteliansky V, Rosenwald A, Basson MA, Licht JD, Pena JT, Rouhanifard SH, Muckenthaler MU, Tuschl T, Martin GR, Bauersachs J, Engelhardt S: Microrna-21 contributes to myocardial disease by stimulating map kinase signalling in fibroblasts. Nature 2008;456:980-984.

-30 Binder BR, Christ G, Gruber F, Grubic N, Hufnagl P, Krebs M, Mihaly J, Prager GW: Plasminogen activator inhibitor 1: Physiological and pathophysiological roles. News Physiol Sci 2002;17:56-61.

-31 Simone TM, Higgins CE, Czekay RP, Law BK, Higgins SP, Archambeault J, Kutz SM, Higgins PJ: Serpine1: A molecular switch in the proliferation-migration dichotomy in wound-"activated" keratinocytes. Adv Wound Care (New Rochelle) 2014;3:281-290.

-32 Hoekstra T, Geleijnse JM, Schouten EG, Kluft C: Plasminogen activator inhibitor-type 1: Its plasma determinants and relation with cardiovascular risk. Thromb Haemost 2004;91:861-872.

-33 Alessi MC, Poggi M, Juhan-Vague I: Plasminogen activator inhibitor-1, adipose tissue and insulin resistance. Curr Opin Lipidol 2007;18:240-245.

-34 Vaughan DE: Pai-1 and atherothrombosis. J Thromb Haemost 2005;3:1879-1883. 